\title{
Análise de aspectos de dependabilidade em infraestruturas de data centers considerando variação de temperatura e diferentes mecanismos de redundância
}

Data centers estão em constante crescimento impulsionando demandas de novas tecnologias tais como computação em nuvem, comércio eletrônico, o que forçou a disponibilização destes sistemas 24 horas por dia, 7 dias por semana sob pena de grandes prejuízos. Em contrapartida, este crescimento tem ocasionado um maior consumo de energia com consequente aumento da dissipação de calor por parte de seus componentes, podendo acarretar elevação da temperatura de operação destes sistemas. Neste trabalho, focamos nos aspectos de dependabilidade de um data center, particularmente considerando a variação da disponibilidade da infraestrutura de comunicação devido à variação de sua temperatura que é proporcionada através da infraestrutura de refrigeração. Com a esta infraestrutura, foram propostas arquiteturas que utilizam diferentes mecanismos de redundância com o objetivo de analisar como ocorre a variação da temperatura quando são utilizadas cada uma destas arquiteturas. Os modelos de dependabilidade foram criados utilizando os mecanismos de modelagem Diagrama de Blocos de Confiabilidade e redes de Petri estocásticas. Além disso, um estudo foi elaborado para a aplicação destes modelos considerando diferentes cenários. Foram feitas diversas análises tais como a verificação da disponibilidade da infraestrutura de comunicação em função da temperatura considerando a variação no tempo de falha de seus componentes, variação da temperatura da infraestrutura de comunicação em função do número de componentes ativos da infraestrutura de refrigeração e as probabilidades de ocorrência de falhas nos componentes da infraestrutura de refrigeração considerando os diferentes mecanismos de redundância adotados.

\section{Analysis of dependability aspects in data center infrastructures considering temperature variation and different redundancy mechanisms}

Data centers are constantly growing, driving demands for new technologies such as cloud computing, e-commerce, which forced the availability of these systems 24 hours a day, 7 days a week under penalty of great losses. On the other hand, this growth has caused a greater consumption of energy with a consequent increase in the heat dissipation by its components, which may lead to an increase in the operating temperature of these systems. In this work, we focus on the dependability aspects of a data center, particularly considering the variation in the availability of the communication infrastructure due to the variation in its temperature that is provided through the cooling infrastructure. With this infrastructure, architectures have been proposed that use different redundancy mechanisms in order to analyze how temperature variation occurs when each of these architectures is used. The dependability models were created using the Reliability Block Diagram modeling mechanisms and stochastic Petri nets. In addition, a study was prepared for the application of these models considering different scenarios. Several analyzes were carried out, such as checking the availability of the communication infrastructure as a function of temperature, considering the variation in the failure time of its components, the variation in the temperature of the communication infrastructure according to the number of active components of the cooling infrastructure and the probabilities failure of components in the cooling infrastructure considering the different redundancy mechanisms adopted.

Keywords: Dependability; Data Center; Temperature Variation; Redundancy Mechanisms.

Topic: Sistemas e Tecnologia da Informação

Reviewed anonymously in the process of blind peer.
Received: 08/06/2020

Approved: 29/07/2020
Almir Pereira Guimarães (ID)

Universidade Federal de Alagoas, Brasil http://lattes.cnpq.br/3181745681718312

http://orcid.org/0000-0002-9242-3355

almirguimaraes@yahoo.com.br

Alan Pereira da Silva (iD)

Universidade Federal de Alagoas, Brasil

http://lattes.cnpq.br/8047275385089590

http://orcid.org/0000-0002-2466-3007

aps@ic.ufal.br
Referencing this:

GUIMARÃES, A. P.; SILVA, A. P.. Análise de aspectos de dependabilidade em infraestruturas de data centers considerando variação de temperatura e diferentes mecanismos de redundância. Revista Brasileira de Administração Científica, v.11, n.3, p.228-241, 2020. DOI: http://doi.org/10.6008/CBPC2179-684X.2020.003.0016 
Análise de aspectos de dependabilidade em infraestruturas de data centers considerando variação de temperatura e diferentes mecanismos de redundância

\section{INTRODUÇÃO}

Durante os últimos anos têm-se notado um crescimento elevado em número, complexidade, tamanho e utilização dos data centers (GUIMARÃES et al., 2015). Desta forma, houve um grande impulso no surgimento de novos paradigmas como computação em nuvem (ARMBRUSTE et al., 2009) e uma grande quantidade de serviços baseados na internet, redes sociais e aplicações multimídia, o que forçou a disponibilização destes sistemas 24 horas por dia, 7 dias por semana sob pena de grandes prejuízos financeiros (MARWAH et al., 2010).

O crescimento elevado dos data centers tem acarretado um maior consumo de energia e um consequente aumento na dissipação de calor por parte de seus componentes. Esta dissipação de calor eleva a temperatura no ambiente do data center podendo provocar uma redução na vida útil de seus componentes e como consequência no funcionamento de suas infraestruturas afetando sua disponibilidade. Este trabalho realiza um estudo relacionado à infraestrutura de comunicação considerando a variação de sua disponibilidade em função da temperatura proporcionada à sala desta infraestrutura. Para proporcionar uma temperatura adequada e constante, diferentes arquiteturas da infraestrutura de refrigeração, caracterizadas por diversos mecanismos de redundância, foram propostas. A falha em qualquer um dos componentes da infraestrutura de refrigeração pode reduzir a capacidade de extração de calor no ambiente da infraestrutura de comunicação e como consequência, resultar em um aumento da temperatura neste ambiente e uma diminuição no tempo de falha e na disponibilidade de seus componentes.

Devido à inexistência do sistema e aos custos de configurações, modelos analíticos são adotados como estratégia para uma análise quantitativa. Com relação à representação dos aspectos de dependabilidade das infraestruturas de comunicação e de refrigeração foram utilizados os mecanismos de modelagem Diagrama de Blocos de Confiabilidade (RBD) (SAHNER et al., 2012) e redes de Petri estocásticas (SPN) (GERMAN, 2000). Serão também aplicadas as equações de energia (SOUZA et al., 2013) e de Arrhenius (ARRHENIUS, 1884; BAYLE et al., 2010) para proporcionar suporte ao cálculo do tempo de falha de cada um dos componentes na infraestrutura de comunicação em função da temperatura proporcionada pela infraestrutura de refrigeração.

Um estudo foi proposto para uma análise, de forma comparativa, do impacto da adoção de diferentes arquiteturas da infraestrutura de refrigeração sobre a variação de temperatura proporcionada aos componentes da infraestrutura de comunicação. Estas arquiteturas caracterizam-se pela composição de diferentes mecanismos de redundância adotados pelos componentes ativos de refrigeração.

Diferentes abordagens tratam da análise do impacto da variação de temperatura em sistemas computacionais. O trabalho mostrado em (BENNACEUR et al., 2018) apresenta um modelo formal para estimar a disponibilidade e a confiabilidade de um sistema de data center em relação ao impacto da variação de temperatura sobre o subsistema de comunicação. Foi utilizado a técnica de modelagem Production Tree (KLOUL et al., 2017) para a modelagem de subsistemas elétricos e térmicos do data center. O trabalho mostrado em (SOUZA et al., 2013) apresenta um conjunto de modelos formais para estimar as perdas 
financeiras em relação ao impacto da variação de temperatura em infraestruturas de comunicação de data centers. Os mecanismos de modelagem RBD (SAHNER et al., 2012) e SPN (GERMAN, 2000) foram adotados para computar a avaliação de dependabilidade. Por sua vez, a Equação de Arrhenius (ARRHENIUS, 1884) e de energia foram utilizadas para o cálculo do tempo de vida dos componentes.

Por sua vez, Patterson (2008) analisa a eficiência energética bem como sugere temperaturas ideais para operação do data center de acordo com suas características. A análise utiliza a técnica CFD (ANDERSON, 1995) para estudo do fluxo de ar na sala. Nesse contexto, os resultados reportados apoiam o desenvolvimento de estratégias eficazes de gerenciamento térmico para os data centers, mas não considera o impacto da variação de temperatura na disponibilidade da infraestrutura de comunicação. Callou et al. (2012) propõe modelos para quantificar o impacto da dependabilidade, sustentabilidade e custos da infraestrutura de refrigeração do data center. A análise das arquiteturas foi feita utilizando modelos SPN e modelos de fluxo de energia (EFM). A partir desses modelos, foram avaliados e encontrados o custo de aquisição, custo operacional, consumo de exergia, downtime e a disponibilidade das arquiteturas de refrigeração. Entretanto, o autor mantém o foco em arquiteturas de refrigeração, não tratando de questões sobre a temperatura.

Por fim, Ghosh et al. (2012) descreve um estudo que analisa a quantidade de servidores que devem ser colocados no interior de um rack, assim como a posição em que devem ser colocadas (por exemplo, na parte superior ou na parte inferior da prateleira). 0 estudo revela que o aumento do número de servidores dentro de um rack tem impacto sobre a temperatura do ar. Este trabalho não foca os efeitos da temperatura sobre a disponibilidade do sistema.

O resto deste artigo é organizado como segue. Seção 2 descreve a metodologia utilizada para a análise dos efeitos da variação de temperatura proporcionada pela infraestrutura de refrigeração sobre a disponibilidade da infraestrutura de comunicação do data center. Seção 3 detalha modelos baseados nos mecanismos de modelagem $R B D$ e $S P N$ representando estruturas comumente utilizadas e adaptações de mecanismos de redundância largamente aplicados em projetos de sistemas. Seção 4 apresenta um estudo aonde foram analisados, de forma comparativa, os efeitos da variação de temperatura proporcionada por diferentes arquiteturas da infraestrutura de refrigeração sobre a disponibilidade da infraestrutura de comunicação de um data center. Finalmente, Seção 6 conclui o artigo e introduz ideias para trabalhos futuros.

\section{METODOLOGIA}

Esta seção detalhada a metodologia concebida para uma análise dos efeitos da variação de temperatura sobre a disponibilidade da infraestrutura de comunicação considerando diferentes arquiteturas da infraestrutura de refrigeração. Nossa abordagem consiste de nove atividades (ver Figura 1): entendimento do problema; obtenção de informações; criação de cenários; criação de modelos; validação de modelos; cálculo da variação de temperatura; cálculo do tempo para falha; avaliação de cenários; interpretação dos resultados. 


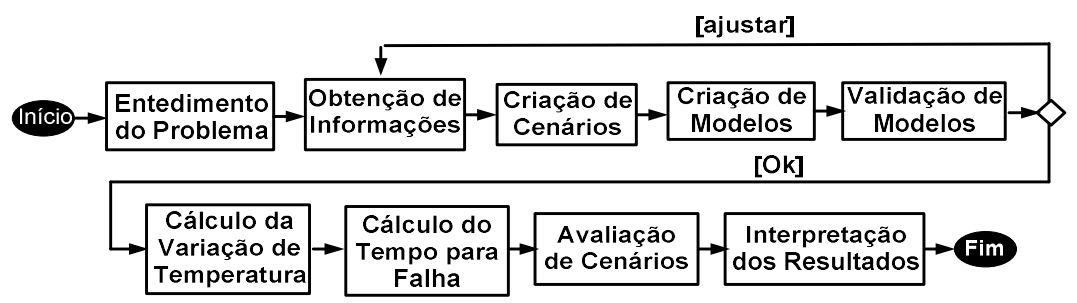

Figura 1: Metodologia Proposta.

A primeira atividade consiste no entendimento do problema. Para executar esta atividade um conjunto de ações devem ser executadas. Dentre as ações podemos destacar: especificação da estrutura do sistema (componentes e interfaces); definição da estrutura e do ambiente (condições de contorno); definição do conjunto de métricas utilizadas para a análise do sistema.

A segunda atividade, obtenção de Informações, trata a respeito da obtenção de informações para os modelos de dependabilidade. Estas informações são oriundas tanto dos fabricantes dos componentes a serem considerados no processo de modelagem, a exemplo do MTTF (sigla proveniente do inglês Mean Time To Failure), como das empresas responsáveis pelas políticas de reparo, tal como o MTTR (sigla proveniente do inglês Mean Time To Repair). Particularmente, com relação a componentes como enlaces, o MTTF pode ser fornecido pela empresa detentora deste recurso, através de uma base de informações.

A terceira atividade, criação de Cenários, trata dos cenários criados através de composições de diferentes arquiteturas das infraestruturas de comunicação e de refrigeração. Então passamos à quarta atividade que é referente à criação de modelos de dependabilidade para o sistema analisado. Com relação a estes modelos, as características do sistema, mecanismos de redundância, nível de interação entre os componentes e as políticas de reparo tratadas irão determinar se iremos utilizar SPN, RBD ou ambos. Esta atividade é executada pela composição de cada componente do sistema de acordo com regras específicas de cada um dos mecanismos de modelagem e pelo mapeamento das métricas desejadas através de expressões representadas pelos elementos de cada modelo. A vantagem da utilização de modelos não baseados em espaço de estados é que eles são eficientes para a especificação e resolução de problemas. Contudo, a solução destes modelos assume a independência dos componentes. Por exemplo, em RBD os componentes devem ser completamente independentes uns dos outros em termos de comportamentos de falha e de reparo. Modelos baseados em espaço de estados, tais como SPN, proporcionam a habilidade de modelar sistemas que violam a suposição feita pelos modelos não baseados em espaço de estados, $R B D$, ao preço da possibilidade de explosão do espaço de estados (BOLCH et al., 2006). Nessa etapa são utilizadas as ferramentas TimeNet (ZIMMERMANN et al., 2000) e Mercury (SILVA et al., 2015) para a construção dos modelos.

A atividade Validação do Modelo analisa e efetua ajustes quando necessário. Para esta atividade, a ferramenta token game (SILVA et al., 2015) e o mecanismo de análise de sensibilidade (BOLCH et al., 2006) são utilizados. O fim desta fase é alcançado quando cada modelo proporciona resultados com exatidão apropriada.

Em seguida, na próxima atividade iremos utilizar o modelo de energia definido em (SOUZA et al., 
2013) (ver Equação 1) para o cálculo da temperatura (TP - expresso em Kelvin) proporcionada ao ambiente da infraestrutura de comunicação em função de diversos fatores. A ferramenta Power Advisor (HEWLETTPACKARD, 2019) foi utilizada para a obtenção de informações tais como consumo de energia e exigências em BTUs (sigla proveniente do inglês British Thermal Unit) a partir dos dispositivos da infraestrutura de comunicação.

$$
T P=\frac{3,413 * P_{C o m} *\left(1-\eta_{C o m}\right) * \Delta t-B T U_{C o m}}{(A r * h)}+\mathrm{TP}_{0}
$$

Aonde temos:

$B T U_{\text {Com }}$ - representa o valor em BTUs proporcionado pela infraestrutura de refrigeração; $P_{\text {Com }}$ (expresso em Watt) - representa a potência instalada da infraestrutura de comunicação; $\mathrm{h}$ (expressa em $\mathrm{W} /\left(\mathrm{m}_{-} \mathrm{K}\right)$ ) - representa a condutividade térmica da parede; $\eta_{\text {Com }}$ - representa a eficiência energética do sistema de energia; $A_{r}$ - representa a área estimada para comportar toda a infraestrutura de comunicação; $\Delta \mathrm{t}$ - representa o período de tempo considerado;

$\mathrm{TP}_{0}$ - representa a temperatura inicial proporcionada à infraestrutura de comunicação. Na próxima atividade, cálculo do tempo para falha, Equação 2 será utilizada para o cálculo do MTTF associado a uma temperatura TP (em Kelvin) de um componente, considerando o MTTF deste componente a uma temperatura inicial TP. Esta equação, baseada na Equação de Arrhenius (ARRHENIUS, 1884; BAYLE et al., 2010) e permite quantificar o impacto da variação de temperatura sobre a tempo para falha do dispositivo da infraestrutura de comunicação através do cálculo de um novo valor de MTTF e o resultado será inserido no respectivo modelo de dependabilidade.

$$
\mathrm{MTTF}_{\mathrm{TP}}=\mathrm{MTTF}_{\mathrm{TPO}} * e^{\left(\frac{-E}{t} *\left(\frac{1}{T P}-\frac{1}{T P 0}\right)\right.}
$$

Aonde temos:
$\mathrm{K}$ - é a constante de Boltzmann $\left(8,623 \times 10^{-5} \mathrm{eV} \mathrm{K}\right)$. $E_{a}$ - é a energia de ativação para ruptura dielétrica de semicondutores; ${ }^{1}$ TPo - é a temperatura inicial ou temperatura desejável proporcionada à infraestrutura de comunicação;

TP - é a temperatura proporcionada à infraestrutura de comunicação em função de diversos fatores. ${ }^{2}$

Na próxima atividade a avaliação de diferentes cenários poderá ser executada. As métricas de interesse podem ser classificadas em duas classes: métricas transientes e métricas estacionárias. Métricas transientes são dependentes do tempo. Por sua vez, métricas estacionárias não dependem de qualquer instante de tempo. Finalmente, os resultados obtidos irão ser interpretados e explicados através da adoção de um vocabulário apropriado.

\section{Modelos de Dependabilidade}

Esta seção apresenta os modelos de dependabilidade utilizados em nosso estudo.

\section{Modelos RBD}

As estruturas mais comumente utilizadas em infraestruturas de sistemas computacionais são série, paralelo e série-paralelo (OGGERINO, 2001). Figura 2 descreve três exemplos, aonde os blocos $\left(c_{1}, c_{2}\right.$ e $\left.C_{3}\right)$

\footnotetext{
${ }^{1}$ A ruptura dielétrica dos semicondutores (gate oxide) utilizada está na faixa de $(0,3 e V-0,7 e V)$ (ON Semiconductor). Este trabalho utiliza um valor de 0,642eV (Vishay).

${ }^{2} \mathrm{Em}$ nosso estudo consideramos TP > TPO.
} 
são estruturados em série (ver Figura 2[a]), em paralelo (ver Figura 2[b]) e em série-paralelo (ver Figura 2[c]).

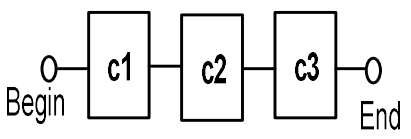

(a) Série

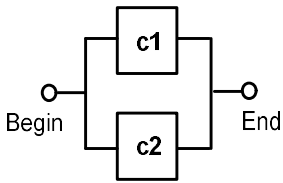

(b) Paralelo

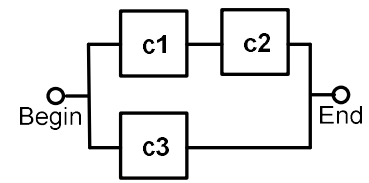

(c) Série - Paralelo

Figura 2: Estruturas Básicas.

$\mathrm{Na}$ estrutura em série, se um componente falhar, o sistema inteiro não estará mais operacional. Assumindo uma estrutura com $n$ componentes em série, a confiabilidade (disponibilidade) (KUO et al., 2003) é obtida por:

$$
P_{(s)}(t)=\prod_{i=1}^{n} P_{i}(\mathrm{t})
$$

Aonde $\mathrm{P}_{\mathrm{i}}(\mathrm{t})$ é a confiabilidade ou a disponibilidade do bloco $i$. Levando-se em consideração $n$ componentes em uma estrutura em paralelo, a confiabilidade (disponibilidade) do sistema é:

$$
\mathrm{P}_{(\mathrm{p})}(\mathrm{t})=\prod_{i=1}^{n}\left(1-P_{i}(t)\right)
$$

Aonde $\mathrm{P}_{\mathrm{i}}(\mathrm{t})$ é a confiabilidade ou a disponibilidade do bloco $i$. Com o objetivo de calcular a confiabilidade (disponibilidade) de uma estrutura série-paralelo, os resultados das equações relacionadas às estruturas em série devem ser combinados e colocados nas equações relacionados às estruturas em paralelo. Para outros exemplos e equações relacionadas, o leitor dever consultar referência (KUO et al., 2003).

\section{Modelos SPN}

Esta seção apresenta os modelos de dependabilidade, que utilizam o mecanismo de modelagem SPN, representando adaptações de mecanismos de redundância largamente utilizados em projetos de sistemas. Estas adaptações serão empregadas para proposição de diferentes arquiteturas da infraestrutura de refrigeração de um data center.

\section{Modular com Reparo e Política de Reposição}

Na Figura 3 mostra o modelo SPN de dependabilidade que representa uma adaptação do mecanismo de redundância denominado de modular (CHIANG et al., 1981) considerando reparo e política de reposição entre seus componentes. Este modelo pode assumir várias configurações de redundância a partir da definição dos valores de $N$ (número de módulos ativos) e de $R$ (número de módulos em espera). Para exemplificar, Figura 3 mostra uma configuração modular $N+R$ que consiste em $N$ módulos ativos e $R$ módulos em espera.

Tabela 1: Parâmetros Transições Imediatas.

\begin{tabular}{|l|l|l|l|}
\hline Transição & Peso & Prioridade & Função de Guarda \\
\hline t0 & - & - & (\#S_ON<=(R-1))AND(\#M_ON=N) \\
\hline t1 & - & - & \#M_ON $<$ N \\
\hline t2 & 0,4 & - & \\
\hline t3 & 0,6 & - & \\
\hline t4 & - & - & \#S_ON=0 \\
\hline t7 & - & - & \#M_ON $<$ N \\
\hline t8 & - & - & (\#S_ON<=(R-1))AND(\#M_ON=N) \\
\hline
\end{tabular}

As $N$ marcações no lugar $M_{-}$ON representam o número de módulos ativos. O número de módulos 
em espera é representado por $R$ no lugar S_ON. Falhas detectadas e falhas não detectadas são representadas pelos lugares MD e MU respectivamente. Foram atribuídas probabilidade de ativação de $40 \%$ e $60 \%$ respectivamente às transições imediatas $t_{2}$ e $t_{3}$ (parâmetro peso na Tabela 1).

Transição MD_MTTR representa o tempo de reparo para falhas detectadas e a Transição MU_MTTR representa o tempo de detecção e reparo para falhas não detectadas de imediato. Com relação às falhas não detectadas, a Transição MU_MTTR tem um acréscimo de 50\% do valor do MTTR em relação às falhas detectadas. A transição imediata $t_{0}$ é ativada quando (\#S_ON<=(R-1)AND\#M_ON=N) e a transição $t_{1}$ é ativada quando (\#M_ON<N). Para a ação de falha detectada, o módulo com defeito é substituído de imediato pelo módulo em espera quando houver módulos em espera disponíveis. Quando o módulo for reparado a transição imediata $t_{7}$ será ativada se $\left(\# \mathrm{M} \_\mathrm{ON}<\mathrm{N}\right)$ devolvendo assim a marcação para o lugar $\mathrm{M} \_\mathrm{ON}$. Se (\#S_ON<=(R-1)AND\#M_ON=N) a transição imediata t 8 será ativada e o módulo reparado será devolvido para o lugar S_ON. Tabela 1 contém os parâmetros das principais transições imediatas deste modelo. A disponibilidade é calculada pela expressão $\mathrm{P}\left\{\# \mathrm{M} \_\mathrm{ON}=\mathrm{N}\right\}$, aonde $N$ é o número de módulos ativos.

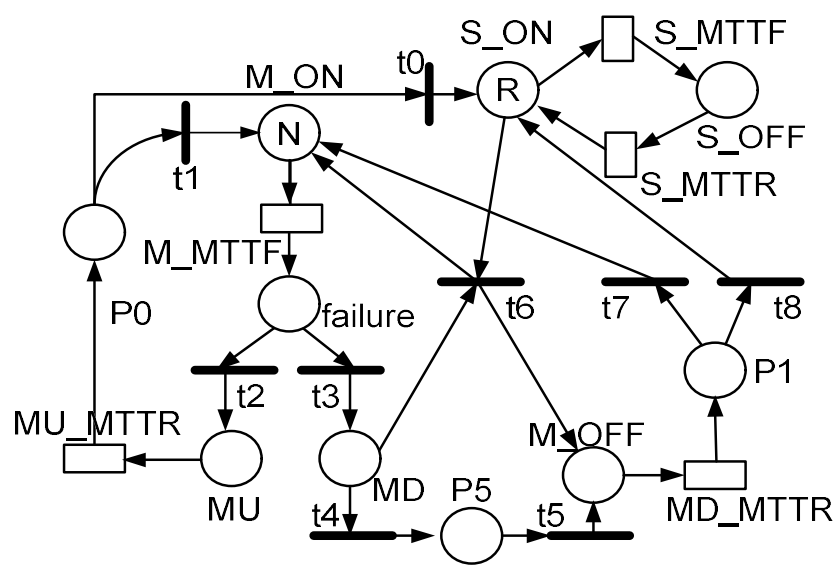

Figura 3: Modelo de Dependabilidade - Modular com Reparo.

\section{Espera a Frio Dupla com Prioridade de Reparo}

Na Figura 4 mostra o modelo SPN que representa uma adaptação do mecanismo de redundância espera a frio (LEE et al., 1990) considerando dois componentes em espera e prioridade de reparo entre eles. Este modelo será denominado de espera a frio dupla com prioridade de reparo. Neste modelo, a marcação no lugar X_ON representa o componente principal no estado ativo e operacional. Dois módulos em espera, representados no lugar Espera, podem ser utilizados em caso de falha do componente principal. Caso este componente falhe, em sua substituição será ativado o módulo 1, representado pela marcação conduzida ao lugar S1_ON pelo disparo das transições Act_Sp e Act_S1. Por sua vez, em caso de falha do componente principal e do módulo 1, será ativado o módulo 2, representado pela marcação conduzida ao lugar S2_ON através do disparo das transições Act_Sp e Act_S2. Em caso de reparo do módulo principal, o estado inicial é reestabelecido. Isto é representado pelo disparo das transições imediatas Dct_S1 e Dct_S2. Tabela 2 contém as funções de guarda que utilizamos nas principais transições imediatas deste modelo. Por sua vez, em caso de falha simultânea do componente principal e dos módulos 1 e 2, a prioridade de reparo será do 
Análise de aspectos de dependabilidade em infraestruturas de data centers considerando variação de temperatura e diferentes mecanismos de

componente principal, do módulo 1 e do módulo 2 nesta ordem. Isto é representado neste modelo pelos arcos inibidores entre o lugar X_OFF e as transições S1_MTTR e S2_MTTR e o arco inibidor entre o lugar S1_OFF e a transição S2_MTTR. A disponibilidade é calculada pela expressão: P\{\#X_ON=1 OR \#S1_ON=1 OR $\left.\# S 2 \_O N=1\right\}$.

Tabela 2: Transições Imediatas - Funções de Guarda.

\begin{tabular}{|l|l|}
\hline Transição & Função de Guarda \\
\hline Act_S1 & \#S1_ON=0 AND \#S1_OFF=0 \\
\hline Act_S2 & \#S1_OFF=1 \\
\hline
\end{tabular}

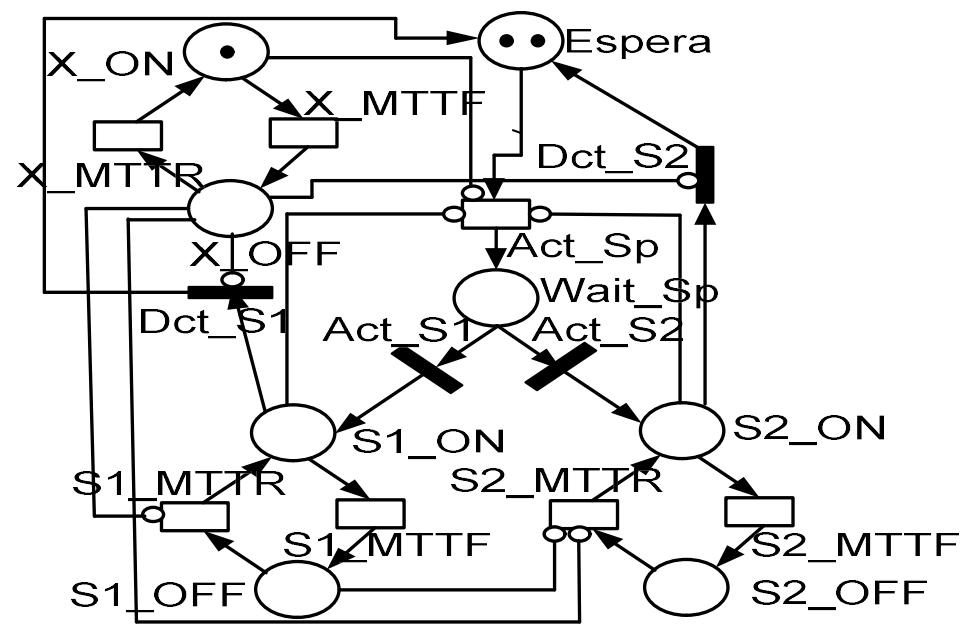

Figura 4: Modelo de Dependabilidade - Espera a Frio Dupla com Prioridade de Reparo.

\section{RESULTADOS E DISCUSSÃO}

Esta parte de nosso estudo tem por objetivo comparar o impacto causado à disponibilidade da infraestrutura de comunicação considerando a variação de temperatura proporcionada por diferentes arquiteturas da infraestrutura de refrigeração em um data center. A infraestrutura de comunicação proposta para o data center é mostrada na Figura 5. Esta infraestrutura adota uma arquitetura com três camadas: núcleo, distribuição e acesso. Cada uma das camadas foi estruturada de forma completamente redundante. As camadas de núcleo e de distribuição adotam o mecanismo de redundância de espera morna (KUO et al., 2003) entre seus componentes.

Particularmente na camada de acesso, a redundância proporcionada pelos switches funciona da seguinte maneira: o switch de acesso S1.2 proporciona redundância ao switch de acesso S1.1; o switch de acesso S1.3 proporciona redundância ao switch de acesso S1.2; continuando nesta ordem, o switch de acesso 1.52 proporciona redundância ao switch de acesso S1.51 e o switch de acesso S1.1 proporciona redundância ao switch de acesso S1.52.

Além disso, cada um dos racks contém dez (10) servidores e cada servidor possui uma completa redundância em relação ao correspondente servidor no próximo rack na sequência, ou seja, o primeiro servidor do rack R1.1 é completamente redundante com o primeiro servidor do próximo rack, R1.2; o segundo servidor do rack R1.1 é completamente redundante com o segundo servidor do rack R1.2. Esta mesma sequência vale para os servidores dos racks R1.3 e rack R1.4, rack R1.5 e rack R1.6, e assim por diante. 
A Figura 6 mostra o modelo RBD de dependabilidade da infraestrutura de comunicação. Os enlaces de fibra e UTP (Unshielded Twisted Pair) que interconectam os roteadores, switches e servidores não foram considerados no processo de modelagem desta infraestrutura. Com relação à infraestrutura de refrigeração, diferentes arquiteturas foram obtidas através da adoção de diversos mecanismos de redundância aplicados a seus componentes. Os modelos de dependabilidade de cada arquitetura proposta para esta infraestrutura adotam uma abordagem hierárquica (TRIVEDI et al., 2009) (ver Figura 7). O nível mais alto corresponde a um modelo $R B D$ com uma estrutura em série e o nível mais baixo corresponde a modelos $S P N$ que representam os mecanismos de redundância associados a componentes do mesmo tipo.

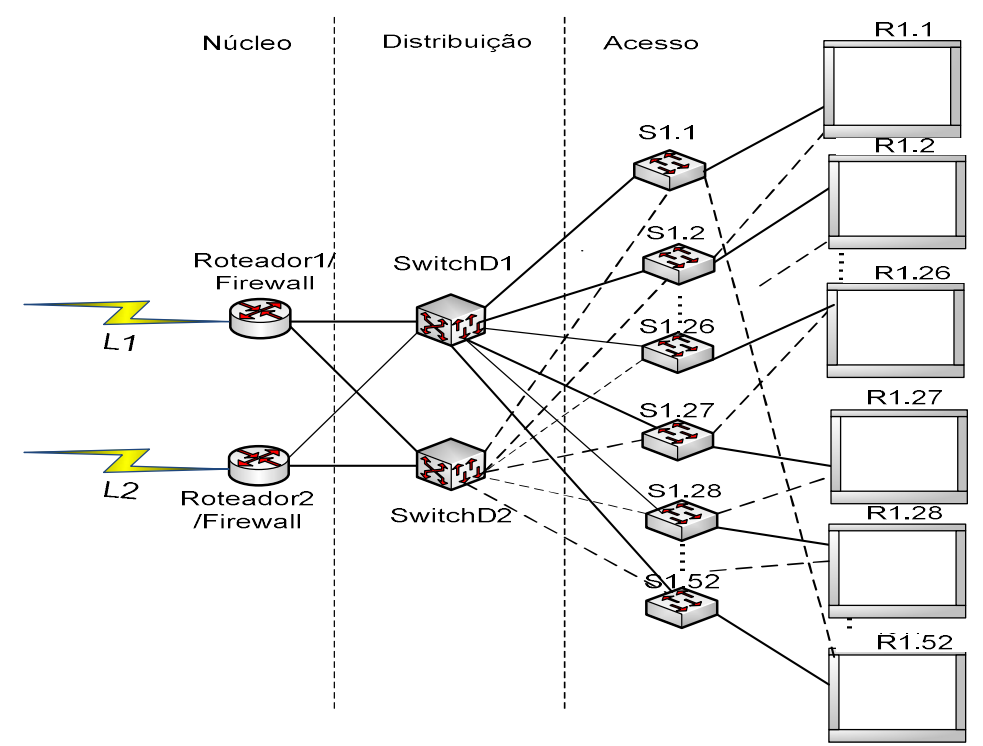

Figura 5: Infraestrutura de Comunicação.

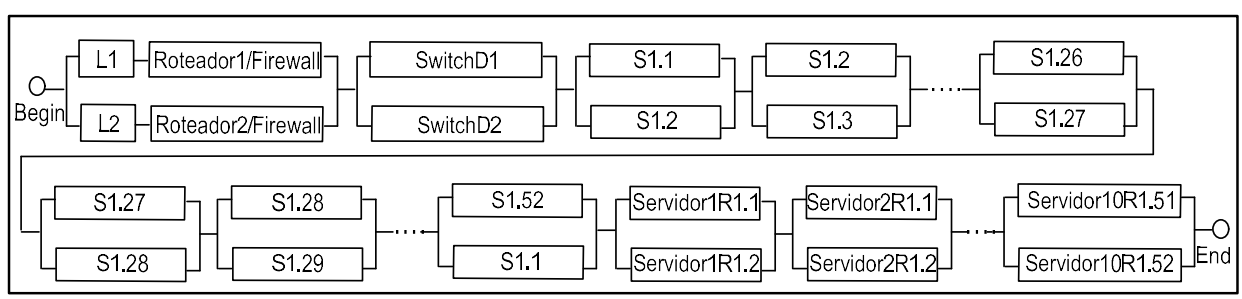

Figura 6: Modelo RBD de Dependabilidade - Infraestrutura de Comunicação.

Particularmente, a Arquitetura A1 (ver Figura 7(a)) utiliza o mecanismo modular com reparo e política de reposição para a redundância entre os componentes CRACs. O mecanismo de redundância de espera a frio (KUO et al., 2003) é utilizado entre os resfriadores. Arquitetura A2 (7[b]) utiliza o mecanismo modular com reparo e política de reposição para a redundância entre os componentes CRACs e o mecanismo Espera Frio Dupla com Prioridade de Reparo para redundância entre os resfriadores.

Seção 3 detalha os modelos SPN de dependabilidade que representam adaptações de mecanismos de redundância largamente utilizados em projetos de sistemas e que foram utilizados nas arquiteturas A1 e A2. Por sua vez, o modelo $S P N$ de dependabilidade que representa o mecanismo de redundância espera a frio foi proposto em (GUIMARÃES et al., 2015).

Tabela 3 mostra os parâmetros de dependabilidade (MTTF e MTTR) relacionados aos componentes das infraestruturas de refrigeração e de comunicação que serão utilizados em seus respectivos modelos de 
dependabilidade. Estes parâmetros foram obtidos em (SILVA et al., 2013; OGGERINO, 2001; KIM et al., 2009).

A Equação 1 (SOUZA et al., 2013) e Equação 2 (ARRHENIUS, 1884; BAYLE et al., 2010) juntamente com os modelos de dependabilidade das infraestruturas de refrigeração e de comunicação, foram utilizadas como base para relacionar o incremento de temperatura com a redução do tempo de falha dos componentes da infraestrutura de comunicação e como consequência em sua disponibilidade.

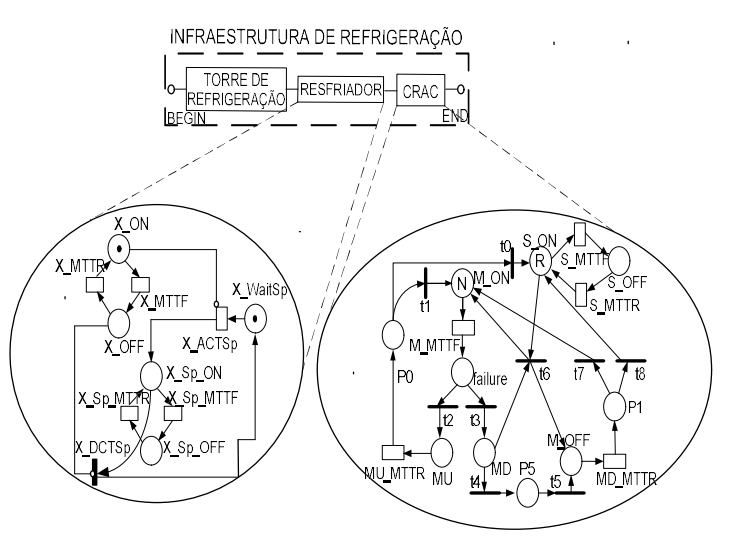

Arquitetura A1

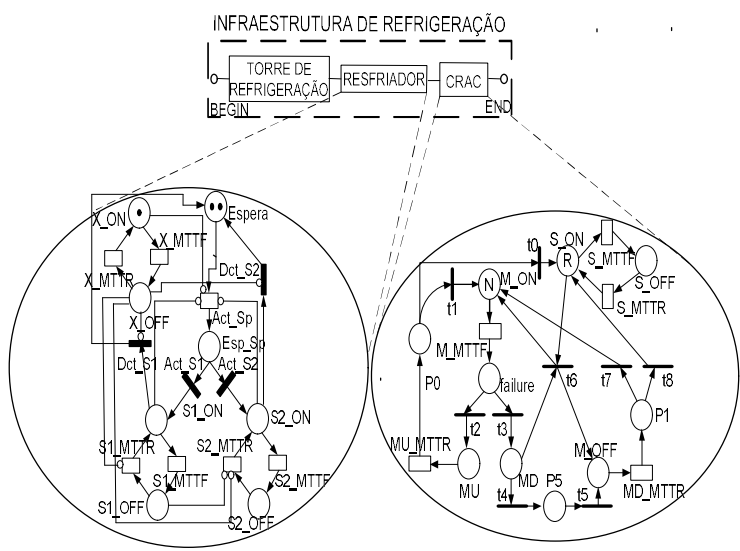

Arquitetura A2

Figura 7: Modelos de Dependabilidade - Infraestrutura de Refrigeração.

Tabela 3: Métricas de Dependabilidade.

\begin{tabular}{|l|l|l|l|l|l|}
\hline Componente & MTTF & MTTR & Componente & MTTF & MTTR \\
\hline Roteador ${ }_{x}$ Firewall & $12.181 \mathrm{~h}$ & $0,52 \mathrm{~h}$ & Switch Acesso ${ }_{x x}$ & $9.090 \mathrm{~h}$ & $0,64 \mathrm{~h}$ \\
\hline Enlace $\left(\mathrm{L}_{1}, \mathrm{~L}_{2}\right)$ & $1.332 \mathrm{~h}$ & $4 \mathrm{~h}$ & Servidor & $1.414 \mathrm{~h}$ & $0,99 \mathrm{~h}$ \\
\hline VMM & $2.880 \mathrm{~h}$ & $1 \mathrm{~h}$ & VM & $2.880 \mathrm{~h}$ & $0,5 \mathrm{~h}$ \\
\hline CRAC & $37.059 \mathrm{~h}$ & $8 \mathrm{~h}$ & Resfriador & $18.000 \mathrm{~h}$ & $48 \mathrm{~h}$ \\
\hline Torre de Refrigeração & $24.816 \mathrm{~h}$ & $48 \mathrm{~h}$ & SwitchD $\mathrm{B}_{\mathrm{X}}$ BalCarga & $12.181 \mathrm{~h}$ & $0,52 \mathrm{~h}$ \\
\hline
\end{tabular}

Inicialmente, foi utilizado a ferramenta Power Advisor para calcular a quantidade de BTUs necessária para proporcionar suporte à infraestrutura de comunicação (ver Figura 5), considerando sua potência instalada (em watts). Foi calculado a necessidade de 312.000 BTUs distribuídos em vinte e seis (26) CRACs. Então, foi aplicada a Equação 1 para o cálculo da temperatura resultante em função da quantidade de BTUs proporcionada pelos CRACs ativos. Figura 8 mostra a variação de temperatura proporcionada ao ambiente da infraestrutura de comunicação em função do número de CRACs ativos. Como esperado, à medida que o número de CRACS diminui, a temperatura resultante irá aumentar. Isso ocorre porque os CRACs não estão produzindo a quantidade de BTUs necessária para o resfriamento adequado da sala da infraestrutura de comunicação do data center.

A partir das temperaturas calculadas em função do número de CRACs foi calculado um novo valor para o parâmetro MTTF de cada um dos componentes da infraestrutura de comunicação utilizando-se a Equação 2. Então, este novo MTTF foi utilizado pelo modelo de dependabilidade desta infraestrutura para quantificar o impacto da variação de temperatura sobre sua disponibilidade.

Figura 9(a) mostra a variação da disponibilidade da infraestrutura de comunicação em função do número de CRACs ativos. Vemos a grande necessidade da utilização de mecanismos de redundância robustos para estes componentes, pois a falha de um CRAC acarreta grande variação da disponibilidade resultante. Por sua vez, Figura 9(b) mostra também a variação da disponibilidade da infraestrutura de comunicação em 
função das temperaturas proporcionadas. Conforme esperado, a disponibilidade decresce consideravelmente com o aumento da temperatura devido à diminuição do parâmetro MTTF calculado segundo Equação 2.

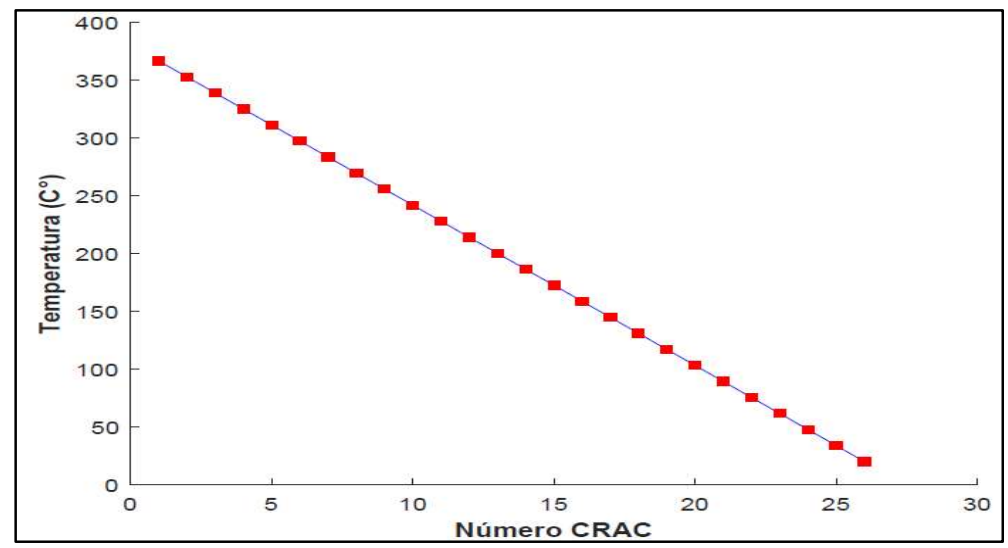

Figura 8: Temperatura X Número CRACs.

Com relação à infraestrutura de refrigeração, eventos de falhas em seus componentes acarreta, devido aos diversos mecanismos de redundância representados nos modelos de dependabilidade mostrados nas Figuras 7(a) (Arquitetura A1) e 7(b) (Arquitetura A2), diferentes temperaturas proporcionadas ao ambiente da infraestrutura de comunicação. Inicialmente, se tivermos um número menor que vinte e quatro (24) CRACs ativos, ou mesmo se um dos componentes torre de refrigeração e resfriador falhar, todo funcionamento da infraestrutura de refrigeração ficará comprometida acarretando uma parada no funcionamento da infraestrutura de comunicação devido às elevadas temperaturas proporcionadas. A probabilidade deste acontecimento é de 0,00193405 para a arquitetura A1 e de 0,00193659 para a arquitetura A2. Devido aos robustos mecanismos de redundância utilizados nas duas arquiteturas, os valores obtidos são próximos. Por fim, Tabela 4 mostra a relação entre temperatura, probabilidade da temperatura e arquiteturas analisadas.

A partir da Equação 1 foi calculada a relação entre número de CRACs ativos e temperatura. Então, utilizamos os modelos de dependabilidade das Arquiteturas A1 e A2 para calcular a probabilidade de funcionamento conjunta dos componentes torre de refrigeração, resfriador e de um determinado número de componentes do tipo CRAC (parâmetro $N$ ). Foram também considerados diferentes quantidades de CRACs em espera (parâmetro $R$ ). Tabela 4 mostra a relação entre a temperatura proporcionada à infraestrutura de comunicação, número de CRACs ativos e probabilidade de funcionamento da infraestrutura de refrigeração nas arquiteturas $\mathrm{A} 1$ e $\mathrm{A} 2$ considerando diferentes quantidades de CRACs em espera (parâmetro $R$ ).

A redução no número de CRACs ativos acarreta uma significativa variação de temperatura proporcionada ao ambiente da infraestrutura de comunicação afetando sua disponibilidade. Devido à utilização de mecanismos de redundância robustos nas Arquiteturas A1 e A2, existe uma probabilidade significativamente maior de que estas arquiteturas operem com o número nominal de CRACs (26) (ver Tabela 4). Por fim, este trabalho buscou a proporcionar uma análise, de maneira comparativa, de como a variação de temperatura proporcionada à sala da infraestrutura de comunicação pode ser influenciada pelos 
Análise de aspectos de dependabilidade em infraestruturas de data centers considerando variação de temperatura e diferentes mecanismos de

diferentes mecanismos de redundância adotados por arquiteturas distintas da infraestrutura de refrigeração.

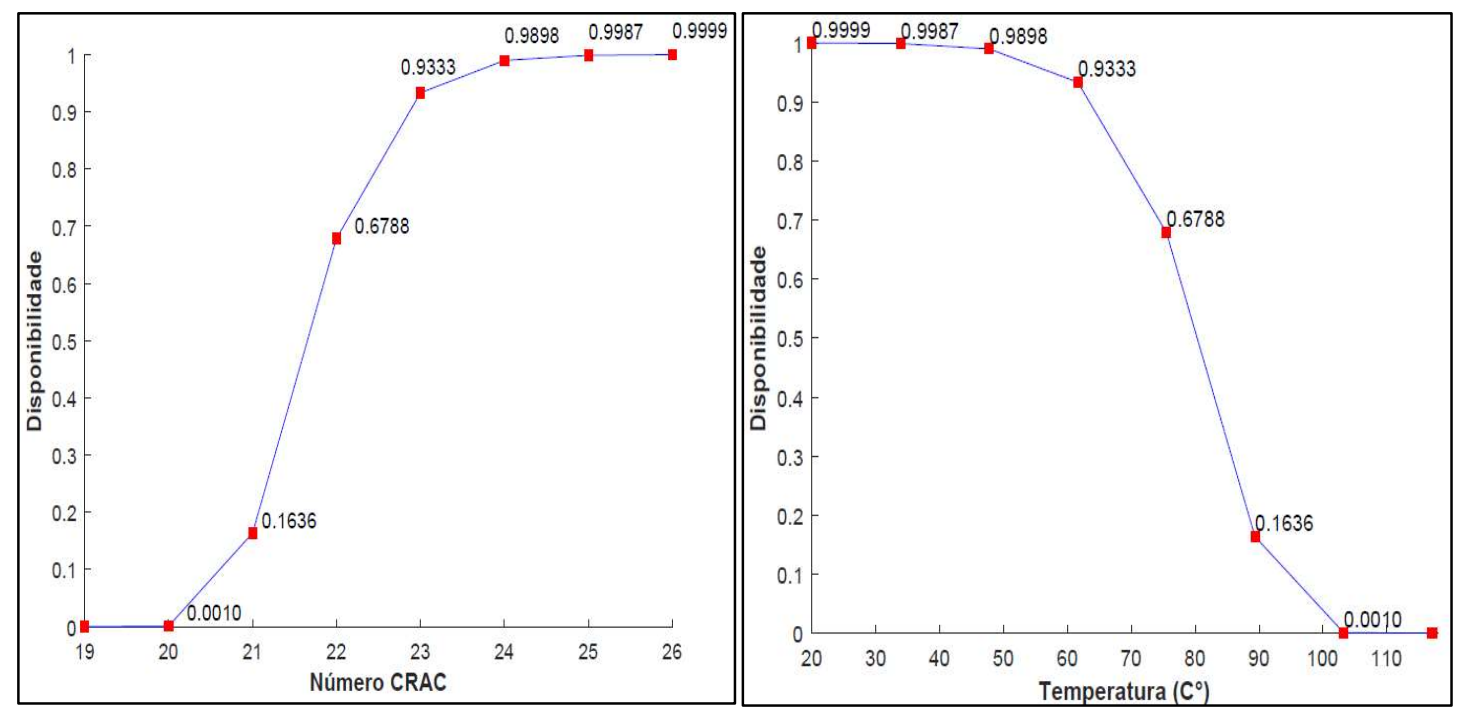

Figura 9: (a) Disponibilidade X Número CRAC; (b) Disponibilidade X Temperatura.

Tabela 4: Relação Número CRACs X Probabilidade Funcionamento Infraestrutura Refrigeração.

\begin{tabular}{|l|l|l|l|l|l|}
\hline Número CRAC & Temperatura & Prob.(A1-R=2) & Prob.(A1-R=1) & Prob.(A2-R=2) & Prob.(A1-R=1) \\
\hline $\mathrm{N}=26$ & $20,00^{\circ} \mathrm{C}$ & 0,99555913 & 0,99553999 & 0,99555541 & 0,99554342 \\
\hline $\mathrm{N}=25$ & $33,87^{\circ} \mathrm{C}$ & 0,0025052 & 0,00251714 & 0,0025052 & 0,00251715 \\
\hline $\mathrm{N}=24$ & $47,75^{\circ} \mathrm{C}$ & 0,0000061 & 0,00000613 & 0,00000606 & 0,00000612 \\
\hline $\mathrm{N}=23$ & $61,62^{\circ} \mathrm{C}$ & 0,00000001414 & 0,00000001435 & 0,00000001409 & 0,00000001435 \\
\hline
\end{tabular}

\section{CONCLUSÕES}

Nosso trabalho propôs modelos analíticos para a análise da disponibilidade da infraestrutura de comunicação de um data center considerando a variação de temperatura em seu ambiente. Para esta variação foi considerado falhas em componentes da infraestrutura de refrigeração. Diferentes mecanismos de redundância foram propostos para os componentes da infraestrutura de refrigeração, considerando diferentes arquiteturas de maneira a analisar a variação da temperatura em cada umas destas. Algumas conclusões podem ser obtidas dos resultados deste estudo.

Primeiro, a variação de temperatura proporcionada pela falha dos componentes da infraestrutura de refrigeração pode ser impeditiva para o funcionamento do data center. Por esta razão mecanismos de redundância robustos devem ser propostos para os diferentes componentes desta infraestrutura. Os custos decorrentes da adoção destes mecanismos podem ser compensados pela alta disponibilidade obtida nas infraestruturas dos data centers. Segundo, combinações dos mecanismos de redundância propostos para a infraestrutura de refrigeração em diferentes arquiteturas acarretou probabilidades diversas de funcionamentos dos componentes CRACs desta infraestrutura. Terceiro, Vale salientar que os modelos SPN de redundância propostos são genéricos o bastante para serem utilizados em diferentes sistemas. Finalmente, para ilustrar a aplicabilidade dos resultados obtidos, um estudo foi realizado destacando o grau de dependência que existe entre o sistema de comunicação e o sistema de refrigeração considerando aspectos de variação de temperatura.

Para trabalhos futuros, podemos aplicar esta abordagem em outros sistemas computacionais. 
Adicionalmente, podemos ampliar os modelos de dependabilidade para incluir diferentes abordagens de redundância em diversas arquiteturas da infraestrutura de refrigeração. Por fim, podemos adotar o mecanismo de análise de sensibilidade para verificar a variação do impacto sobre a métrica disponibilidade a partir de cada parâmetro dos modelos de dependabilidade da infraestrutura de refrigeração em função da variação de temperatura.

\section{REFERÊNCIAS}

ANDERSON, J. D.. Computational fluid dynamics: The basics with applications. 1995.

ARMBRUST, M.; FOX, A.; GRIFFITH, R.; JOSEPH, A.D.; KATZ, R.; KONWINSKI, A.; LEE, G.; PATTERSON, D.; RABKIN, A.; ZAHARIA, M.. Above the clouds: A Berkeley view of cloud computing. University of California at Berkeley UCB/EECS. 2009.

ARRHENIUS, S.. Recherches sur la conductibilité galvanique des électrolytes. 1884.

BAYLE, F.; METTAS, A.. Temperature acceleration models in reliability predictions: Justification \& improvements. Annual Reliability and Maintainability Symposium (RAMS), 2010. DOI: http://doi.org/10.1109/RAMS.2010.5448028

BENNACEUR, W. M.; KLOUL, L.. Electrical and thermal system impact on the availability of a data center's system. In: INTERNATIONAL CONFERENCE ON SYSTEM RELIABILITY AND SAFETY, 3. Anais. ICSRS, 2018. p.142-148. DOI: http://doi.org/10.1109/ICSRS.2018.8688831

BOLCH, G.; GREINER, S.; MEER, H.; TRIVEDI, K. S.. Queuing networks and markov chains: Modelling and performance evaluation with computer science applications. 2 ed. 2006.

CALLOU, G.; MACIEL, P.; TUTSCH, D.; ARAÚJO, J.. Models for dependability and sustainability analysis of data center cooling architectures. In: INTERNATIONAL CONFERENCE ON DEPENDABLE SYSTEMS AND NETWORKS WORKSHOPS (DSN). Anais. IEEE/IFIP, 2012. p.1-6. DOI:

http://doi.org/10.1109/DSNW.2012.6264697

CHIANG, D. T.; NIU, S.. Reliability of consecutive-k-out-of-n: F-system. IEEE Transactions on Reliability, v.R30, n.1, p.8789, 1981. DOI: http://doi.org/10.1109/TR.1981.5220981

GERMAN, R.. Performance analysis of communicating systems - modeling with non - markovian stochastic petri nets. 2000 .

GHOSH, R.; SUNDARALINGAM, V.; JOSHI, Y.. Effect of rack server population on temperatures in data centers. In: INTERSOCIETY CONFERENCE ON THERMAL AND THERMOMECHANICAL PHENOMENA IN ELECTRONIC SYSTEMS, 13. Anais. 2012. p.30-37. DOI: http://doi.org/10.1109/ITHERM.2012.6231410

GUIMARÃES, A. P.; MACIEL, P.; MATIAS JUNIOR, R.. Design of it infrastructures of data centers: An approach based on business and technical metrics. Quantitative Assessments of Distributed Systems. Performability Engineering Series, 2015.
HEWLETT-PACKARD. Hewlett-Packard Company. HPE Power Advisor: A tool for estimating power requirements for HPE enterprise solutions. Hewlett-Packard, 2019.

KIM, D. S.; MACHIDA, F.; TRIVEDI, K. S.. Availability modeling and analysis of a virtualized system. In: PACIFIC RIM INTERNATIONAL SYMPOSIUM ON DEPENDABLE COMPUTING, 15. Anais. 2009. p.365-371. DOI: http://doi.org/10.1109/PRDC.2009.64

KLOUL, L.; RAUZY, A.. Production trees: a new modeling methodology for production availability analyses. Reliability Engineering \& System Safety, v.167, p.561-571, 2017. DOI: http://doi.org/10.1016/i.ress.2017.06.017

KUO, W.; ZUO, M. J.. Optimal reliability modeling: Principles and applications. 2003.

LEE, P. A.; ANDERSON, T.. Fault tolerance: Principles and practice. 2 ed. 1990.

MARWAH, M.; MACIEL, P.; SHAH, A.; SHARMA, R.; CHRISTIAN, T.; ALMEIDA, V.; ARAÚJO, C., SOUSA, E.; CALLOU, G.; SILVA, B.; GALDINO, S.; PIRES, J.. Quantifying the sustainability impact of data center availability. SIGMETRICS Performance Evaluation Review, v.37, p.64-68, 2010. DOI: http://doi.org/10.1145/1773394.1773405

OGGERINO, C.. High availability network fundamentals. 2001.

PATTERSON, M.. The effect of data center temperature on energy efficiency. In: INTERSOCIETY CONFERENCE ON THERMAL AND THERMOMECHANICAL PHENOMENA IN ELECTRONIC SYSTEMS, 11. Anais. 2008. p1167-1174. DOI: http://doi.org/10.1109/ITHERM.2008.4544393

SAHNER, R.; TRIVEDI, K. S.; PULIAFITO, A.. Performance and reliability analysis of computer systems: an example-based approach using the Sharpe software package. 2012.

SILVA, B.; CALLOU, G.; TAVARES, E.; MACIEL, P.; FIGUEIREDO, J.; SOUSA, E.; ARAÚJO, C.; MAGNANI, F.; NEVES, F.. Astro: An integrated environment for dependability and sustainability evaluation. Sustainable Computing: Informatics and Systems, v.3, n.1, p.1-17, 2013. DOI: http://doi.org/10.1016/j.suscom.2012.10.004

SILVA, B.; MATOS, R.; CALLOU, G.; FIGUEIREDO, J.; OLIVEIRA, D.; FERREIRA, J.; DANTAS, J.; ALVES, V.; MACIEL, P.. Mercury: An integrated environment for performance and dependability evaluation of general system. In: DEPENDABLE 
Análise de aspectos de dependabilidade em infraestruturas de data centers considerando variação de temperatura e diferentes mecanismos de redundância

SYSTEMS AND NETWORKS CONFERENCE, 45. Anais. DSN, 2015.

SOUZA, R.; CALLOU, G.; CAMBOIN, K.; FERREIRA, J.; MACIEL, $P$.. The effects of temperature variation on data center IT systems. In: IEEE INTERNATIONAL CONFERENCE ON SYSTEMS, MAN, AND CYBERNETICS. Anais. SMC, 2013. p.2354-2359. DOI: http://doi.org/10.1109/SMC.2013.402

TRIVEDI, K. S.; KIM, D. S.; ROY, A.; MEDHI, D.. Dependability and security models. In: INTERNATIONAL WORKSHOP ON
DESIGN OF RELIABLE COMMUNICATION NETWORKS, 7. Anais. 2009. p.11-20. DOI:

http://doi.org/10.1109/DRCN.2009.5340029

ZIMMERMANN, A.; FREIHEIT, J.; GERMAN, R.; HOMMEL, G. Petri net modelling and performability evaluation with time net 3.0. In: COMPUTER PERFORMANCE EVALUATION. MODELLING TECHNIQUES AND TOOLS: INTERNATIONAL CONFERENCE, 11. Anais. Schaumburg, 2000. p.188-202.

A CBPC - Companhia Brasileira de Produção Científica (CNPJ: 11.221.422/0001-03) detém os direitos materiais desta publicação. Os direitos referem-se à publicação do trabalho em qualquer parte do mundo, incluindo os direitos às renovações, expansões e disseminações da contribuição, bem como outros direitos subsidiários. Todos os trabalhos publicados eletronicamente poderão posteriormente ser publicados em coletâneas impressas sob coordenação da Sustenere Publishing, da Companhia Brasileira de Produção Científica e seus parceiros autorizados. Os (as) autores (as) preservam os direitos autorais, mas não têm permissão para a publicação da contribuição em outro meio, impresso ou digital, em português ou em tradução. 